\title{
Philadelphia chromosome with acute myeloid leukemia and concurrent large $B$ cell lymphoma of different origins: A case report
}

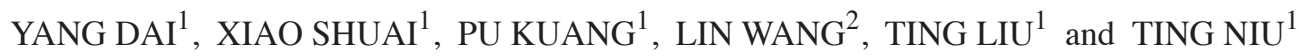 \\ Departments of ${ }^{1}$ Hematology and Research Laboratory of Hematology and ${ }^{2}$ Pathology, West China Hospital, \\ Sichuan University, Chengdu, Sichuan 610041, P.R. China
}

Received January 20, 2015; Accepted March 3, 2016

DOI: $10.3892 / \mathrm{ol} .2017 .5578$

\begin{abstract}
Philadelphia chromosome with de novo acute myeloid leukemia $(\mathrm{Ph}+\mathrm{AML})$ arising from $\mathrm{t}(9 ; 22)$ is an uncommon occurrence. $\mathrm{Ph}+\mathrm{AML}$ is known to respond poorly to conventional chemotherapy. To the best of our knowledge, simultaneous diagnosis of de novo $\mathrm{Ph}+\mathrm{AML}$ and lymphoma in a single patient has not yet been reported. The present study reports the case of a 37-year-old female patient who presented with bone pain, fever and lymphadenopathy, and was diagnosed as $\mathrm{Ph}+\mathrm{AML}$ with concurrent diffuse large B cell lymphoma. Combined chemotherapy regimen covering AML and lymphoma was administered, achieving short-term response. However, the therapy soon failed and the patient succumbed to the disease. The present study reports the first case of $\mathrm{Ph}+\mathrm{AML}$ occurring concurrently with diffuse large B cell lymphoma, and discusses certain differences between $\mathrm{Ph}+\mathrm{AML}$ and chronic myelogenous leukemia in the myeloid blast crisis phase, as well as the appropriate therapeutic modalities for $\mathrm{Ph}+\mathrm{AML}$. In addition, the potential association between $\mathrm{Ph}+\mathrm{AML}$ and diffuse large $\mathrm{B}$ cell lymphoma in this patient was investigated.
\end{abstract}

\section{Introduction}

The Philadelphia chromosome $(\mathrm{Ph})$ or breakpoint cluster region-Abelson $(B C R-A B L)$ fusion gene is more common in patients with chronic myelogenous leukemia (CML) (1) than in those with precursor B-acute lymphoblastic leukemia

Correspondence to: Dr Yang Dai or Dr Ting Niu, Department of Hematology and Research Laboratory of Hematology, West China Hospital, Sichuan University, 37 Guo Xue Xiang Street, Chengdu, Sichuan 610041, P.R. China

E-mail: danshine77@163.com

E-mail: tingniu1@sina.com

Key words: Philadelphia chromosome + acute myeloid leukemia, large B cell lymphoma, tyrosine kinase inhibitor, therapy
(ALL) (2); however, $\mathrm{Ph}+$ acute myeloid leukemia (AML) has also been reported.

AML has an incidence of 3.7 per 100,000 individuals, with anemia, bleeding, fever and bone pain as its typical symptoms. Currently, the standard therapy for AML consists of chemotherapy, immunotherapy, targeting therapy and hematopoietic stem cell transplantation $(3,4)$.

Although AML secondary to chemotherapy or radiotherapy has been previously reported (5), to the best of our knowledge, there are no reports of concurrent $\mathrm{Ph}+\mathrm{AML}$ and B cell lymphoma in untreated patients. The current case reports presents a patient who presented with leukocytosis and lymphadenopathy and was diagnosed with $\mathrm{Ph}+$ AML concurrent with large B cell lymphoma.

\section{Case report}

A 37-year-old Chinese woman complaining of bone pain and fever was admitted to the West China Hospital of Sichuan University (Chengdu, China) in June 2011. The patient was previously healthy, and the complete blood count (CBC) at 3 months prior to admission was normal. Upon pathological examination, multiple bone tenderness and a painless $2 \times 1-\mathrm{cm}$ lymph node in the left axillary fossa were palpable. Laboratory test results suggested mild leukocytosis $\left(13.6 \times 10^{6} / \mu \mathrm{l}\right.$; normal range, $3.5-9.5 \times 10^{6} / \mu 1$ ) with $10 \%$ white blood cells but normal hemoglobin and platelet counts. Bone marrow smear demonstrated $33.5 \%$ myeloid blasts and biopsy revealed diffuse infiltration of AML-like cells. Flow cytometry (FCM) results demonstrated white blood cells co-expressing human leukocyte antigen-antigen D-related, CD34, CD38, CD13, CD33, CD117, cytoplasmic myeloperoxidase and aberrant CD19. Giemsa-banding discovered $\mathrm{t}(9 ; 22)(\mathrm{q} 34 ; \mathrm{q} 11)$ in $5 / 20$ metaphases (Fig. 1). Quantitative polymerase chain reaction (PCR) detected P210 type $B C R-A B L$ fusion gene and $B C R-A B L / A B L$ ratio as $5.45 \times 10^{5}$ copies $/ \mathrm{ml}(5.19 \%)$. Bone scan with ${ }^{99 \mathrm{~m}} \mathrm{Tc}-\mathrm{meth}-$ ylene diphosphonate revealed multiple abnormalities throughout the body. The BCR-ABL fusion gene RT-PCR detection kit was purchased from Shanghai Source Biomedical Technology Co., Ltd. (Shanghai, China). The thermocycling conditions were as follows: $42^{\circ} \mathrm{C}$ for $30 \mathrm{~min}, 94^{\circ} \mathrm{C}$ for $5 \mathrm{~min}$, $94^{\circ} \mathrm{C}$ for $15 \mathrm{sec}$ and $60^{\circ} \mathrm{C}$ for $60 \mathrm{sec}$, for a total of 40 cycles. An 


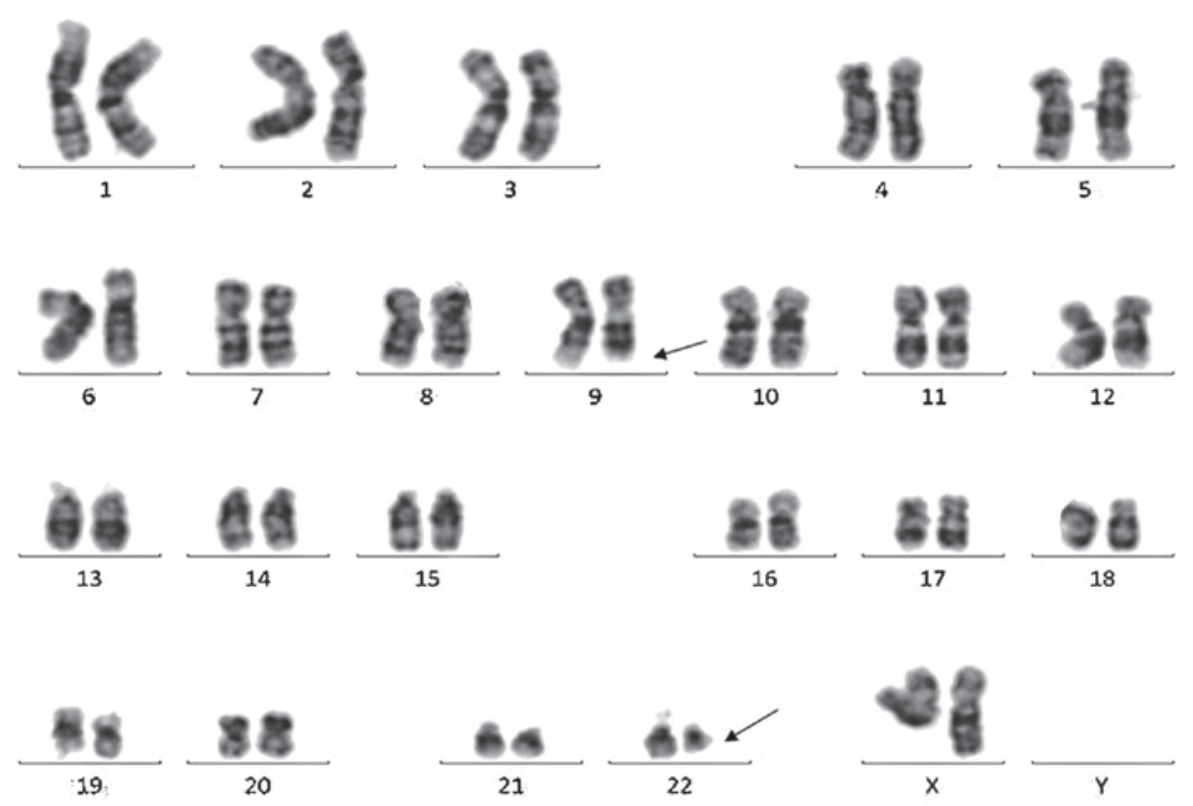

Figure 1. Conventional cytogenetic analysis with Giemsa-banding detected the translocation of $\mathrm{t}(9 ; 22)(\mathrm{q} 34 ; \mathrm{q} 11)$ in $5 / 20$ metaphases (arrow).

$1.5 \times 1.5-\mathrm{cm}$ lymph node in the left axillary fossa was revealed by computed tomography (CT) scan. Lymph node biopsy was performed and the formalin-fixed, paraffin-embedded tissue was subjected to pathological diagnosis. The results supported the diagnosis of non-Hodgkin lymphoma (diffuse large B cell lymphoma, aggressive, of non-germinal center B cell origin) according to the 2008 World Health Organization Classification (Fig. 2) (4). Considering the leukemic cells harboring the $B C R-A B L$ gene, fluorescence in situ hybridization (FISH) with $B C R-A B L$ probes was performed on the lymph node specimen with no fusion signal detected (Fig. 3). Two days later, the patient developed a headache on the right side of the head, without nausea or vomiting. Head CT scan was normal, while T2-weighted magnetic resonance imaging scan demonstrated a long, contrast agent-intensified signal on multiple sites of the skull, excluding the cerebral parenchyma. The results of routine, biochemical and FCM tests of the cerebrospinal fluid were normal.

Based on the aforementioned findings, $\mathrm{Ph}+\mathrm{AML}$ with concurrent diffuse large $\mathrm{B}$ cell lymphoma was diagnosed. The bone pain and headache were considered to indicate leukemia or lymphoma infiltration, due to no evidence of infection. Treatment with rituximab (600 $\mathrm{mg}$ on day 1$)$, adriamycin (70 mg on day 2), cyclophosphamide (1,000 mg on day 2), vincristine (2 $\mathrm{mg}$ on day 2 ), cytarabine (150 $\mathrm{mg}$ on days $2-8$ ) and dexamethasone (15 mg on days 2-8) was administered and relieved the symptoms shortly. The lymph node returned to its normal state and complete hematologic remission was obtained; however, $B C R-A B L$ transcripts remained positive. Two courses of a similar regimen followed for consolidation. CHR was confirmed prior to each consolidation, while $B C R-A B L$ transcripts remained detectable and increased ( $B C R-A B L / A B L$, from 0.67 up to $8.8 \%$ ). Soon after the second consolidation, the patient developed middle grade fever and joint pain in the left knee; leukemia relapse was confirmed by bone marrow smear, FCM and further elevated $B C R-A B L$ transcripts (BCR-ABL/ABL, 71.73\%). Treatment with imatinib mesylate (IM) (400 mg daily for $>2$ months) and chemotherapy [10 mg idarubicin (days 1,3 and 5), $2 \mathrm{mg}$ vincristine (day 1), $100 \mathrm{mg}$ cytarabine (days 1-5) and $30 \mathrm{mg}$ prednisone (days 1-5)] was administered, alleviating the symptoms from the following day; however, the reappearing and continuously increasing peripheral blasts suggested leukemia progression. IM was considered ineffective and the bone marrow specimen was sent to confirm the suspected $A B L$ kinase mutation, which demonstrated $F 395 \mathrm{~V}$ mutation. For that purpose, DNA was extracted from the bone marrow specimen, and PCR and DNA sequencing were used to assess the presence of the F395V mutation (Fig. 4). In addition, gene mutations common for AML (CEBPA, DNMT3A, FLT3-ITD, IDH1, IDH2, KIT, KRAS, NPM1, NRAS, TET2 and WT1) were investigated in smears at diagnosis and relapse and were all negative. Due to ineffectiveness, IM was switched for dasatinib (75 mg daily), which resulted in a mild decrease in $B C R$ - $A B L$ transcripts, but no hematologic response. Twenty days after relapse, the patient suddenly developed an intracranial hemorrhage and succumbed shortly after.

Written informed consent was signed by the husband of the patient and approved by the Ethics Committee of the West China Hospital of Sichuan University (Chengdu, China).

\section{Discussion}

The Ph chromosome or $B C R-A B L$ fusion gene can be found in $>95 \%$ of all CML cases, and $5-30 \%$ of adult and $2-5 \%$ of pediatric ALL cases (2); however, it has also been reported in AML. Keung et al (6) conducted a retrospective study of 148 cases of $\mathrm{t}(9 ; 22)(\mathrm{q} 34 ; \mathrm{q} 11)$, and identified $84 \%$ as $\mathrm{CML}$ chronic phase, $13 \%$ as de novo ALL, $1 \%$ as de novo AML and $2 \%$ as myelodysplastic syndrome (MDS). The estimated incidence of $\mathrm{Ph}+\mathrm{AML}$ was $0.6 \%$. Ph + AMLs were reported with either major or minor BCR gene rearrangements, similar to those of $\mathrm{Ph}+\mathrm{ALL}$, suggesting that $\mathrm{Ph}+\mathrm{AML}$ is a distinct disease rather than CML-myeloid blast crisis (MBC) phase. 


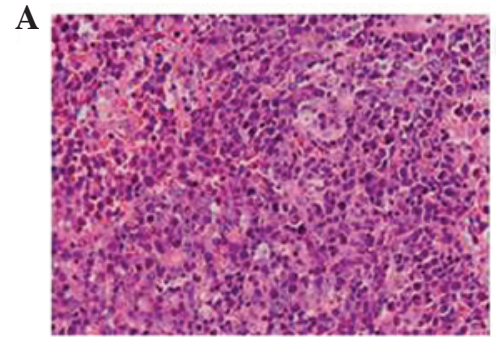

D

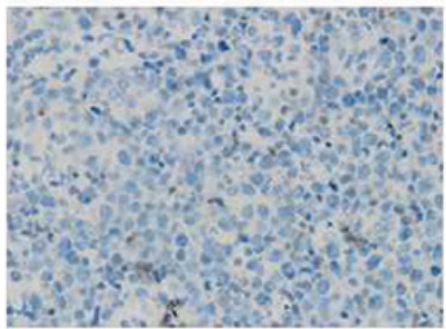

G

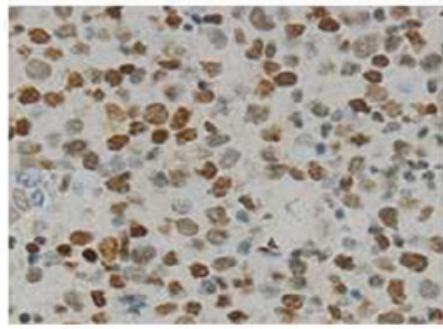

B

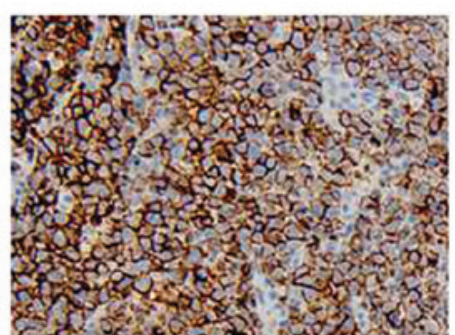

$\mathbf{E}$
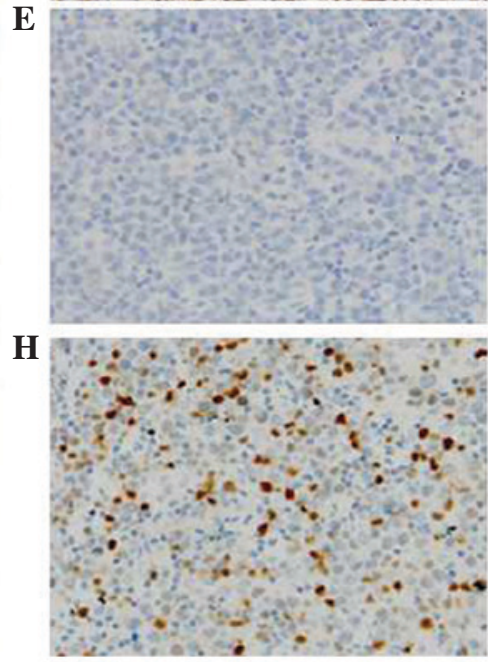

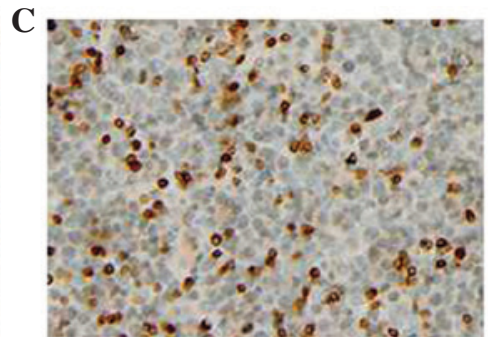

$\mathbf{F}$
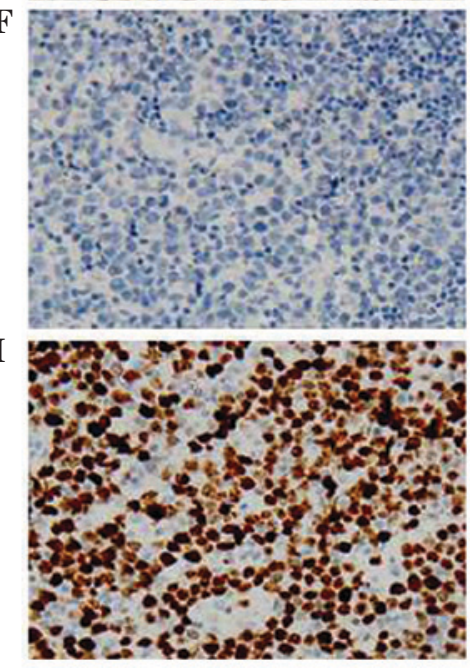

Figure 2. H\&E and IHC staining of the specimens from the involved lymph node, supporting the diagnosis of diffuse large B cell lymphoma. (A) H\&E staining. IHC staining showing positivity for and negativity for (B) cytomembrane expression of CD20. (C) CD3e, (D) CD5, (E) ALK-1 and (F) CD30. Cytoplasmic expression of (G) B-cell lymphoma 6 and $(\mathrm{H})$ interferon regulatory factor 4. (I) The percentage of Ki-67-positive cells was $~ 80 \%$. Magnification, x200. H\&E, hematoxylin \& eosin; IHC, immunohistochemical.

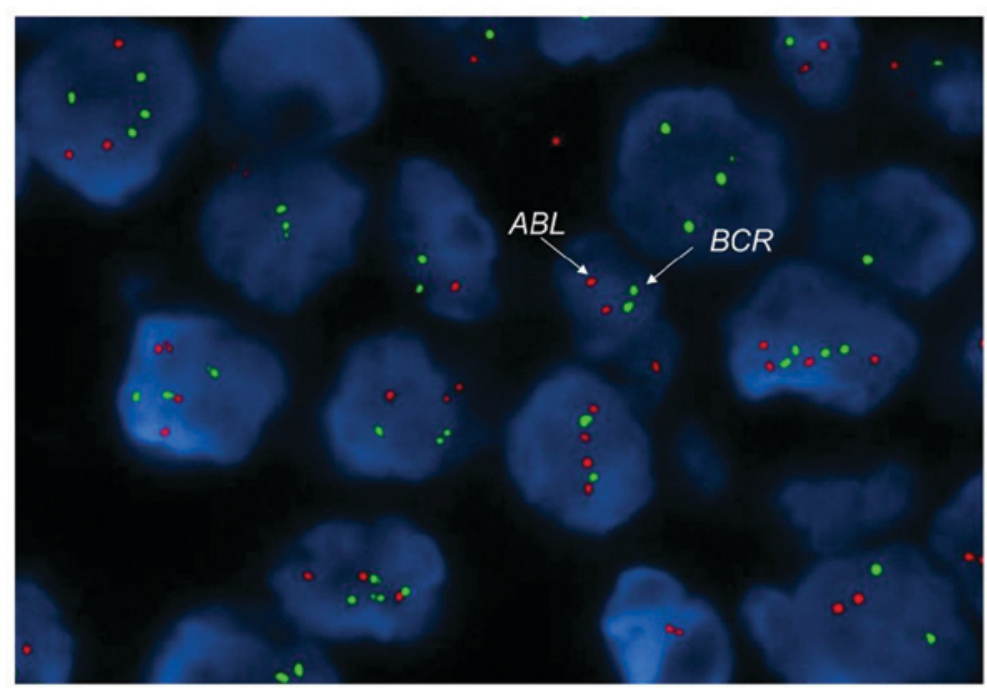

Figure 3. Fluorescence in situ hybridization with BCR-ABL probes was performed on the lymphoma specimen, and no BCR-ABL fusion signal was detected (red, ABL; green, BCR; magnification, x100). BCR, breakpoint cluster region; ABL, Abelson.

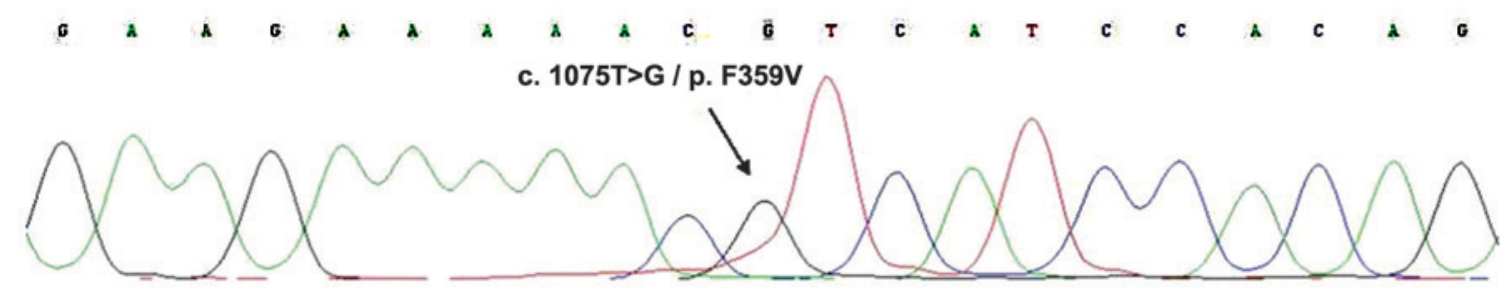

Figure 4. Abelson kinase gene mutation analysis during Philadelphia chromosome + acute myeloid leukemia relapse revealed the existence of the F395V mutation (arrow). 
Furthermore, rare cases of $\mathrm{Ph}+\mathrm{MDS}$ were also reported, which imply that $\mathrm{Ph}+\mathrm{AML}$ is a distinct disease entity rather than representing blastic transformation from CML (6). Clinically, $\mathrm{Ph}+\mathrm{AML}$ presents with less incidence of splenomegaly and significant basophilia (7). Immunophenotypic analysis of $\mathrm{Ph}+\mathrm{AML}$ disclosed co-expression of CD34 and multiple myeloid markers (such as CD13 and CD33), and a common aberrant expression of lymphoid markers $(\geq 2$ in $60 \%$ of cases) (8); additional cytogenetic abnormalities, such as extra copies of $\mathrm{Ph}$ or trisomy 8 , were more commonly detected in CML-MBC, compared with Ph + AML (59.9 vs. $25 \% ; \mathrm{P}=0.008$ ) (9). The coexistence of normal and $\mathrm{Ph}^{+}$metaphases at diagnosis is more characteristic of Ph + AML (5). The induction of chemotherapy usually causes $\mathrm{CBC}$ and karyotype normalization in $\mathrm{Ph}+\mathrm{AML}$, but chronic or accelerated-phase hemogram and persistence of the $\mathrm{Ph}$ chromosome in CML-MBC $(7,8)$. Konoplev et al $(10)$ detected NPM1 and FLT3-ITD mutations (frequent in AML) in 2/9 and 1/9 of patients with $\mathrm{Ph}+\mathrm{AML}$, respectively, in addition to no mutation in patients with CML-MBC, and no $A B L 1$ mutation (common in CML-MBC) in any of the $9 \mathrm{Ph}+$ AML patients. Array comparative genomic hybridization was performed in $\mathrm{Ph}+\mathrm{AML}$, bilineage leukemia, $\mathrm{Ph}+\mathrm{ALL}$ and CML (11). Losses of IGH, TRG2, VPREBI and IGLL1 were detected in $\mathrm{Ph}+\mathrm{AML}, \mathrm{Ph}+\mathrm{ALL}$ and CML lymphoid BC but not in CML-chronic phase, CML-MBC or AML with normal karyotype, which further verified the difference between $\mathrm{Ph}+\mathrm{AML}$ and CML-MBC; however, no single clinical or laboratory test can definitively distinguish the two diseases. The previous $\mathrm{CBC}$ of the present patient was normal, and no splenomegaly and basophilia were found at diagnosis. The FCM test result suggested a diagnosis of $\mathrm{Ph}+\mathrm{AML}$. Following the induction of chemotherapy, the $\mathrm{CBC}$ returned to normal. Taken together, all aforementioned findings suggest that de novo $\mathrm{Ph}+\mathrm{AML}$ may have been a more appropriate diagnosis for this patient.

Due to its rarity, no standard therapies for $\mathrm{Ph}+\mathrm{AML}$ have been established, and treatment options derive largely from reports of similar cases. In the pre-tyrosine kinase inhibitor (TKI) era, Ph + AML was usually treated by conventional chemotherapy. Cuneo et al (9) reported that conventional chemotherapy achieved CR in 4/11 patients (36\%), while in the study by Paietta $e t$ al, none of the 6 patients obtained remission (5). Due to its marked effect on CML, IM was also used in $\mathrm{Ph}+\mathrm{AML}$. IM was reported for the treatment of $\mathrm{Ph}+\mathrm{AML}$, achieving sustained cytogenetic response and 1 case of molecular remission for 15 months (12-14). In a larger retrospective treatment study, 7/16 patients were treated with IM, among which, 6 achieved HR and 1 achieved CHR, although the response durations were short (median, 2.5 months; range, 1-6 months) (9). IM should therefore be considered front-line therapy (9), but the optimal dosage, timing and duration remain to be determined.

IM is usually administered at a dose of $400 \mathrm{mg}$ daily, when combined with chemotherapy $(15,16)$, or at a dose of $600 \mathrm{mg}$ daily when administered alone (12-14). Sun et al (17) reported 2 patients with $\mathrm{Ph}+\mathrm{AML}$, who were treated with IM and daunorubicin-based chemotherapy, followed by allo-hematopoietic stem cell transplantation (HSCT) and IM maintenance regimen. Both patients obtained CHR and complete cytogenetic and molecular responses for 44 and
48 months. Another patient receiving unrelated allo-HSCT during CR2, and sustained CHR for 70 months (18); therefore, IM combined with chemotherapy, followed by allo-HSCT and IM maintenance treatment appears to be an effective treatment option for $\mathrm{Ph}+\mathrm{AML}$, particularly when IM is used early (17).

IM was not initially administered to the present patient, which may account for the failure of molecular response. Although IM was added following relapse, it failed to bring molecular effect due to the $F 395 \mathrm{~V}$ mutation. Despite dasatinib treatment theoretically overcoming this mutation, desatinib may have been used too late and led only to a decrease in transcripts but no HR. This finding implies that the BCR-ABL signal is not the only or not the major pathway to cause $\mathrm{Ph}+\mathrm{AML}$. The initial treatment plan for the present patient was treatment with allo-HSCT, but it was hindered by the lack of matched donor. In addition, auto-HSCT could not be considered for a patient without molecular remission.

Despite the fact that secondary AML or MDS has been frequently reported in patients receiving chemotherapy and/or radiotherapy, concomitant lymphoma and AML in previously untreated patients is extremely rare (19). Only a few cases of AML concurrent with lymphoma have been reported (19-23); To the best of our knowledge, this is the first report of simultaneous Ph + AML and diffuse large B cell lymphoma. It is known that leukemia may involve extramedullary sites, including lymph nodes. In the present case, the lymph node biopsy revealed no leukemic cells, excluding AML infiltration of lymph nodes. In addition, lymphoma may disseminate to the bone marrow resembling leukemia; however, no lymphoma cells were detected by FCM in the bone marrow specimen, excluding lymphoma dissemination as well. Since CML may progress to both myeloid and lymphoid leukemia, it has been hypothesized that a single pluripotent stem cell may develop either myeloid or lymphoid neoplasms (24). It was therefore assumed that the leukemia and lymphoma in the present patient may have had the same origin. The leukemic cells were shown to harbor the Ph chromosome, but no $B C R-A B L$ fusion signal was observed on the lymph node specimen, indicating that the two diseases were unlikely to share the same origin. One hypothesis is that the single stem cell gained different second hits (mutations) during differentiation and developed distinct neoplasms. Cauwelier et al (25) detected monoclonal B lymphocytes in the blood and marrow of a patient with MDS (with trisomy 13), but no evidence of lymphoma. FISH was performed on sorted $\mathrm{CD} 19^{+}$and $\mathrm{CD} 34^{+}$cells for the detection of trisomy 13. Trisomy 13 was detected in $55 \%$ of CD $34^{+}$cells and $5.5 \%$ of $\mathrm{CD}^{+} 9^{+}$cells, the latter was considered negative. $\mathrm{X}$-chromosome inactivation showed that both $\mathrm{CD} 34^{+}$and $\mathrm{CD} 19^{+}$cells were monoclonal, while their inactivated chromosomes were different, suggesting that the two populations had different origins.

The etiology of dual tumors such as the one in the present case were obscure. The patient had worked as a nurse in a dental clinic for a long period of time. It was unclear whether the extended exposure to dental repairing materials had played a role in the development and pathogenesis of the disease. Jaalouk et al (22) reported a case of concurrent large B cell lymphoma and MDS, whose treatment with steroids caused a rapid augmentation of the myeloid clone and transformation to AML, indicating that the lymphoid clone may downregulate 
the myeloid clone. In the present patient, the enlarged lymph node was normalized soon after chemotherapy treatment and remained normal thereafter, while the leukemia deteriorated rapidly, supporting the interclonal inhibition hypothesis.

$\mathrm{Ph}+\mathrm{AML}$ is a rare disease associated with poor prognosis. It possesses different clinical manifestations and laboratory test results from those of CML-MBC, and should therefore be regarded as a distinct disease. Appropriate treatment options include TKI, combined chemotherapy and treatment with allo-HSCT. Although AML or MDS secondary to chemotherapy, radiotherapy and/or HSCT have previously been reported, $\mathrm{Ph}+\mathrm{AML}$ concurrent with large B cell lymphoma is considerably more rare, and its underlying pathogenesis remains to be elucidated.

\section{References}

1. Faderl S, Talpaz M, Estrov Z, O'Brien S, Kurzrock R and Kantarjian HM: The biology of chronic myeloid leukemia. N Engl J Med 341: 164-172, 1999.

2. Fielding AK: How I treat Philadelphia chromosome-positive acute lymphoblastic leukemia. Blood 116: 3409-3417, 2010.

3. Deschler B and Lübbert M: Acute myeloid leukemia: Epidemiology and etiology. Cancer 107: 2099-2107, 2006.

4. Vardiman JW, Thiele J, Arber DA, Brunning RD, Borowitz MJ, Porwit A, Harris NL, Le Beau MM, Hellström-Lindberg E, Tefferi A and Bloomfield CD: The 2008 revision of the World Health Organization (WHO) classification of myeloid neoplasms and acute leukemia: Rationale and important changes. Blood 114: 937-951, 2009.

5. Paietta E, Racevskis J, Bennett JM, Neuberg D, Cassileth PA, Rowe JM and Wiernik PH: Biologic heterogeneity in Philadelphia chromosome-positive acute leukemia with myeloid morphology: The eastern cooperative oncology group experience. Leukemia 12: 1881-1885, 1998.

6. Keung YK, Beaty M, Powell BL, Molnar I, Buss D and Pettenati M: Philadelphia chromosome positive myelodysplastic syndrome and acute myeloid leukemia-retrospective study and review of literature. Leuk Res 28: 579-586, 2004.

7. Berger R: Differences between blastic chronic myeloid leukemia and Ph-positive acute leukemia. Leuk Lymphoma 11 (Suppl 1): 235-237, 1993

8. Soupir CP, Vergilio JA, Dal Cin P, Muzikansky A, Kantarjian H, Jones D and Hasserjian RP: Philadelphia chromosome-positive acute myeloid leukemia: A rare aggressive leukemia with clinicopathologic features distinct from chronic myeloid leukemia in myeloid blast crisis. Am J Clin Pathol 127: 642-650, 2007.

9. Cuneo A, Ferrant A, Michaux JL, Demuynck H, Boogaerts M, Louwagie A, Doyen C, Stul M, Cassiman JJ, Dal Cin P, et al: Philadelphia chromosome-positive acute myeloid leukemia: Cytoimmunologic and cytogenetic features. Haematologica 81: 423-427, 1996.

10. Konoplev S, Yin CC, Kornblau SM, Kantarjian HM, Konopleva M, Andreeff M, Lu G, Zuo Z, Luthra R, Medeiros LJ and Bueso-Ramos CE: Molecular characterization of De novo Philadelphia chromosome-positive acute myeloid leukemia. Leuk Lymphoma 54: 138-144, 2013.
11. Nacheva EP, Grace CD, Brazma D, Gancheva K, Howard-Reeves J, Rai L, Gale RE, Linch DC, Hills RK, Russell N, et al: Does BCR/ABL1 positive acute myeloid leukaemia exist? Br J Haematol 161: 541-550, 2013

12. Ito K, Tominaga K, Suzuki T, Jinnai I and Bessho M: Successful treatment with imatinib mesylate in a case of minor BCR-ABL-positive acute myelogenous leukemia. Int J Hematol 81: 242-245, 2005.

13. Jentsch-Ullrich K, Pelz AF, Braun H, Koenigsmann M, Mohren M, Wieacker P and Franke A: Complete molecular remission in a patient with Philadelphia-chromosome positive acute myeloid leukemia after conventional therapy and imatinib. Haematologica 89: ECR15, 2004.

14. Yamaguchi M and Konishi I: Successful treatment with imatinib mesylate for Philadelphia chromosome-positive refractory acute myeloid leukemia. Rinsho Ketsueki 44: 254-256, 2003.

15. Lazarevici V, Golovleva I, Nygren I and Wahlin A: Induction chemotherapy and post-remission Imatinib therapy for De novo BCR-ABL positive AML. Am J Hematol 81: 470-471, 2006.

16. Kondo T, Tasaka T, Sano F, Matsuda K, Kubo Y, Matsuhashi Y, Nakanishi H, Sadahira Y, Wada H, Sugihara T and Tohyama K: Philadelphia chromosome-positive acute myeloid leukemia $(\mathrm{Ph}$ +AML) treated with imatinib mesylate (IM): A report with IM plasma concentration and bcr-abl transcripts. Leuk Res 33: e137-e138, 2009

17. Sun J, Wang Z, Luo Y, Tan Y, Allan DS and Huang H: Prolonged survival with imatinib mesylate combined with chemotherapy and allogeneic stem cell transplantation in De novo $\mathrm{Ph}+$ acute myeloid leukemia. Acta Haematol 127: 143-148, 2012.

18. Bacher U, Haferlach T, Alpermann T, Zenger M, Hochhaus A, Beelen DW, Uppenkamp M, Rummel M, Kern W, Schnittger S and Haferlach C: Subclones with the $t(9 ; 22) / B C R-A B L 1$ rearrangement occur in AML and seem to cooperate with distinct genetic alterations. Br J Haematol 152: 713-720, 2011.

19. Kapadia SB and Kaplan SS: Simultaneous occurrence of non-Hodgkin's lymphoma and acute myelomonocytic leukemia. Cancer 38: 2557-2560, 1976.

20. Youness E, Ahearn MJ and Drewinko B: Simultaneous occurrence of non-Hodgkin's lymphoma and spontaneous acute granulocytic leukemia. Am J Clin Pathol 70: 415-420, 1978.

21. Ohwada C, Nakaseko C, Tanaka H, Abe D, Oda K, Ozawa S, Takeuchi M, Shimizu N, Cho R, Saito Y and Nishimura M: Successful matched unrelated BMT for secondary AML which developed simultaneously with relapsed Hodgkin's lymphoma. Bone Marrow Transplant 39: 569-570, 2007.

22. Jaalouk G, Avvisati G, Latagliata R, Pacchiarotti A, Pulsoni A, Mecarocci S and Malagnino F: Simultaneous occurrence of large B-cell non-Hodgkin lymphoma and myelodysplastic syndrome rapidly evolving into acute myeloblastic leukemia. Leuk Lymphoma 21: 339-341, 1996.

23. Montefusco E, Fazi F, Cordone I, Ariola C, Nanni M, Spadea A, Spiriti MA, Fenu S, Mandelli F and Petti MC: Molecular remission following high-dose hydroxyurea and fludarabine plus cytarabine in a patient with simultaneous acute myeloid leukemia and low-grade lymphoma. Leuk Lymphoma 40: 671-674, 2001.

24. Janossy G, Woodruff RK, Paxton A, Greaves MF, Capellaro D, Kirk B, Innes EM, Eden OB, Lewis C, Catovsky D and Hoffbrand AV: Membrane marker and cell separation studies in Ph1-positive leukemia. Blood 51: 861-877, 1978.

25. Cauwelier B, Nollet F, De Laere E, Van Leeuwen M, Billiet J, Criel A and Louwagie A: Simultaneous occurrence of myelodysplastic syndrome and monoclonal B lymphocytes with a different clonal origin. Leuk Lymphoma 43: 191-193, 2002. 